\title{
Predictors of Mortality in Patients of Acute Exacerbation of Chronic Obstructive Pulmonary Disease: A Prospective Observational Study
}

\author{
Hemant Kumar, Satyadeo Choubey ${ }^{1}$ \\ Department of Respiratory Medicine, Dr. Ram Manohar Lohia Institute of Medical Sciences, Lucknow, Uttar Pradesh, ${ }^{1}$ Department of Respiratory Medicine, MGM \\ Medical College, Indore, Madhya Pradesh, India
}

\section{Abstract}

Background: Acute exacerbations of chronic obstructive pulmonary disease (AECOPD) result in increased morbidity, mortality and tremendous socioeconomic burden. Predicting inhospital mortality may aid prognostication, planning for the site of care, i.e., ward versus intensive care units, and better individualization of treatment. Aim of Study: This study aims in predicting inhospital mortality in patients of AECOPD based on the parameters measured at the time of admission. Methods: Known COPD patients in acute exacerbations admitted in a tertiary care hospital were interrogated for clinical history and examination. All relevant laboratory tests including arterial blood gas analysis, complete blood count, liver and renal function tests, and random blood sugar were done. Based on outcome, patients were grouped into survivors and nonsurvivors. Parameters recorded were then subjected for univariate analysis to get their statistical significance. All significant variables on univariate analysis were then analyzed further with multivariate analysis. Results: Out of the total 140 patients included in the study, $24(17 \%)$ died during their hospital stay. Of the various acute-phase parameters recorded at the time of the admission, only five qualified to be predictors of inhospital mortality based on univariate and multivariate analyses. These were partial pressure of carbon dioxide in arterial blood $\left(\mathrm{PaCO}_{2}\right)$ (odds ratio [OR], 95\% confidence interval [CI] $\left.=1.067,0.993-1.146\right)$, pH $(\mathrm{OR}, 95 \% \mathrm{CI}=0.001,0.001-0.584)$, serum glutamic pyruvate transaminase $(\mathrm{SGPT})(\mathrm{OR}, 95 \% \mathrm{CI}=1.032,1.006-1.059)$, sodium $(\mathrm{OR}, 95 \% \mathrm{CI}=0.779,0.689-0.881)$, and random blood sugar $(\mathrm{OR}, 95 \% \mathrm{CI}=1.018,1.007-1.029)$. With these five factors combined, area under receiver operating characteristic (ROC) curve was 0.9684 , sensitivity $79.18 \%$, specificity $96.55 \%$, positive predictive value $82.61 \%$, negative predictive value $95.73 \%$, and correctly classify acute exacerbation in $93.57 \%$. Good survival can be expected if these parameters are within normal limits. Conclusion: $\mathrm{pH}, \mathrm{PaCO}, \mathrm{SGPT}$, serum sodium, and random blood sugar at the time of admission are independent predictors of mortality in patients of AECOPD. These can be helpful in developing a prediction tool of inhospital mortality in such patients.

Keywords: Acute exacerbation of chronic obstructive pulmonary disease, chronic obstructive pulmonary disease, predictors of mortality

\section{INTRODUCTION}

Chronic Obstructive Pulmonary Disease (COPD) is a chronic disease involving the airways, lung parenchyma, and pulmonary vasculature and also has considerable systemic manifestations. The disease is progressive with possible gene-environment interaction and hence can be prevented by avoiding exposure to the noxious particles. The most common studied attributing risk factor is cigarette smoking in any form. Exacerbations and comorbidities play their role in contributing to overall severity. ${ }^{[1]}$

The disease is one of the most important causes of death worldwide. The Global Burden of Disease Study projected it

\begin{tabular}{|l|l|}
\hline \multicolumn{2}{|c|}{ Access this article online } \\
\hline Quick Response Code: & Website: \\
\hline & www.ijrconline.org \\
\hline
\end{tabular}

to become the third leading cause of death globally by the year 2020 , while a newer projection estimated it to be the fourth one by $2030 .{ }^{[2]} \mathrm{An}$ exacerbation is an acute event in patients of COPD with increased baseline respiratory symptoms requiring additional treatment other than the usual ongoing treatment. ${ }^{[1]}$ These events have negative effects on the patient's quality of life, accelerate the decline in lung function, are associated

Address for correspondence: Dr. Satyadeo Choubey, Department of Respiratory Medicine, MGM Medical College, Indore, Madhya Pradesh, India E-mail: chestsd@gmail.com

This is an open access journal, and articles are distributed under the terms of the Creative Commons Attribution-NonCommercial-ShareAlike 4.0 License, which allows others to remix, tweak, and build upon the work non-commercially, as long as appropriate credit is given and the new creations are licensed under the identical terms.

For reprints contact: reprints@medknow.com

How to cite this article: Kumar H, Choubey S. Predictors of mortality in patients of acute exacerbation of chronic obstructive pulmonary disease: A prospective observational study. Indian J Respir Care 2018;7:77-82. 
with significant mortality, and pose a great socioeconomic burden. ${ }^{[3-5]}$

In hospitalized patients, the mortality is approximately $10 \%$ during the hospital stay. It may go up to $40 \%$ during the $1^{\text {st }}$ year of discharge from the hospital in mechanically ventilated patients. The all-cause mortality during the 3 years of hospitalization is as high as $49 \% .^{[5,6]}$ Therefore, prompt management of exacerbations, early detection, and prevention are crucial in the overall management of COPD patients.

In developing countries like India, the direct attributable medical cost seems less appealing figure wise, but there is tremendous impact of COPD on both workplace and home productivity. The disease may force two individuals to leave the workplace for each case, i.e., the affected individual and a healthy family member to take care of the disability of the former at home.

Identifying the patients depending on the factors predicting mortality is of utmost importance to reduce the poor outcome of exacerbation. There are many indices which can predict mortality in stable COPD patients such as BODE index and ADO index, but only few scoring systems are available for acute exacerbation of chronic obstructive pulmonary disease (AECOPD) such as DECAF score (the dyspnea, eosinopenia, consolidation, acidaemia, and atrial fibrillation) and BAP-65 (blood urea nitrogen, altered mental status, pulse $>109$ beats/min, and age $>65$ years)..$^{[7,8]}$

Hence, the present study is intended to identify the possible predictors or parameters at the time of admission which can help predict the mortality in AECOPD.

\section{Methods}

This prospective, observational study was conducted in Department of Respiratory Medicine, Sir Sunderlal Hospital, Institute of Medical Sciences, Banaras Hindu University, Varanasi. A total of 140 eligible patients were recruited.

All patients who had been admitted for an AECOPD during April 2010 to June 2011 were included. An exacerbation was defined by the presence of an increase in at least one of the following three symptoms: dyspnoea, cough, sputum amount and/or purulence severe enough to warrant hospital admission. All patients were documented cases of COPD previously confirmed with pulmonary function test (forced expiratory volume in one second/forced vital capacity $<0.7$ and irreversible airway obstruction) and were receiving a combination of various bronchodilators. During hospital admission, patients were treated with a standard protocol consisting of intravenous administration of corticosteroids $(0.5 \mathrm{mg} / \mathrm{kg})$, theophylline, nebulized salbutamol, and ipratropium bromide. Oxygen therapy was titrated according to the results of blood gas analyses and pulse oximetry.

Patients who presented with exacerbation of COPD without any documentary evidence of COPD before present event, known
COPD mimics such as bronchial asthma, bronchiectasis, congestive heart failure, those with comorbidities such as known diabetes, renal disease, hepatic disease, neurological disease, coronary artery disease, patients with multiple organ failure, hemodynamic instability, and those patients who were not giving consent were excluded.

All eligible patients underwent detailed history, clinical examination, and relevant investigations such as complete blood count, renal and liver function tests, random blood sugar, hemoglobin A1c, X-ray chest posteroanterior view, electrocardiogram, echocardiography, and arterial blood gas (ABG) analysis .

All patients were managed primarily according to Global Initiative for Chronic Obstructive Lung Disease guideline protocol with other required conservative management, for example, raised blood sugar was managed with insulin with target value of $180 \mathrm{mg} / \mathrm{dl}$. Based on outcome after hospital stay, patients were divided into two groups:

Group 1 (Nonsurvived or Expired) - This group includes those patients who died during hospital stay.

Group 2 (Survived) - This group includes all those patients who survived during hospital stay and discharged to home.

All demographic, clinical, and laboratory parameters were compared between these two groups.

\section{Statistical methods}

Discrete data were analyzed by cross-tables using descriptive method. Continuous data were analyzed by univariate analysis. Univariate and multivariate analysis to derive hazard ratio was done using Cox proportional hazard model. $P<0.05$ was considered as statistically significant association.

\section{RESULTS}

A total of 140 patients were enrolled, out of which 116 survived and 24 patients died during the hospital stay. Hence, the overall inhospital mortality rate for AECOPD was 17\% [Figure 1]. Actual mortality may have been high if comorbidities such as ischemic heart disease and congestive heart failure were also included.

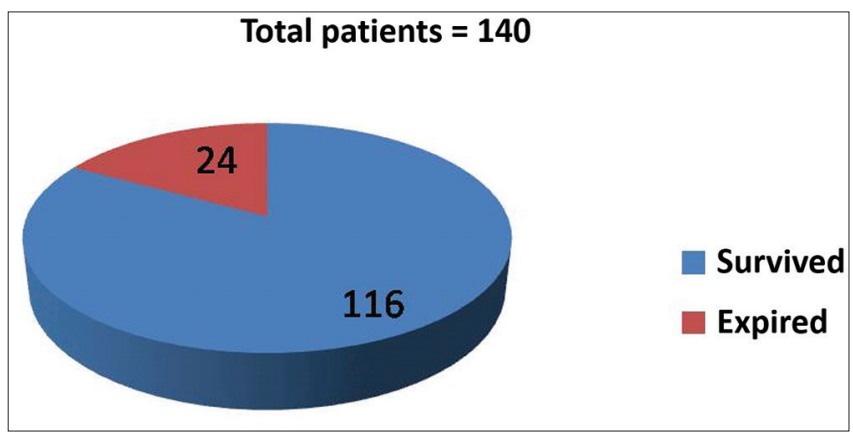

Figure 1: Survival rate in patients with chronic obstructive pulmonary disease admitted with acute exacerbation 
The mean ( \pm standard deviation) age of the patients who survived was significantly lower $(P=0.046)$ at $61.24( \pm 8.228)$ years as against those who died during hospital stay $65.62( \pm 5.830)$ years [Figure 2]. There were 30 females and 110 male participants in this study [Figure 3]. Males outnumbered females by almost four times [Figure 3]. It may be partly because of the fact that smoking is more prevalent in males and women tend to present later in the course of the disease [Table 1].

Ninety-two patients $(65.7 \%)$ in the study were smokers, mostly male, indicating that smoking is an important risk factor for COPD. History of previous hospitalization due to an acute episode was more in the nonsurviving group (75\%) as compared to surviving (59\%) depicting an elevated mortality rate (odds ratio 2) [Table 1].

\section{Univariate analysis}

Important laboratory parameters at the time of admission were analyzed by univariate analysis [Table 2].

In the $A B G$, the partial pressure of carbon dioxide in arterial blood $\left(\mathrm{PaCO}_{2}\right)$ was significantly high $(72.08 \mathrm{mmHg}$ vs. $53.23 \mathrm{mmHg}, P<0.05)$ and $\mathrm{pH}$ significantly low

\begin{tabular}{lccc}
\hline \multicolumn{4}{l}{ Table 1: Demographic data of all patients } \\
\hline \multicolumn{1}{l}{$\boldsymbol{n}$} & Survived & Expired \\
\hline Total patients (\%) & 140 & $116(83)$ & $24(17)$ \\
Age (years), mean \pm SD & & $61.24 \pm 8.23$ & $65.62 \pm 5.83$ \\
Sex & & & \\
$\quad$ Male & 110 & 91 & 19 \\
$\quad$ Female & 30 & 25 & 5 \\
Smoking habits & & & \\
$\quad \begin{array}{l}\text { Smoker } \\
\text { Nonsmoker }\end{array}$ & 92 & 73 & 19 \\
$\begin{array}{l}\text { Number of pack years } \\
\text { (mean } \pm \text { SD) }\end{array}$ & 48 & 43 & 5 \\
$\begin{array}{l}\text { Prior hospital admission, } \\
n(\%)\end{array}$ & & $18.21 \pm 8.44$ & $23.83 \pm 4.22$ \\
$\begin{array}{l}\text { Total duration of years } \\
\text { (mean } \pm \text { SD) }\end{array}$ & & $68(59)$ & $18(75)$ \\
\hline
\end{tabular}

SD: Standard deviation

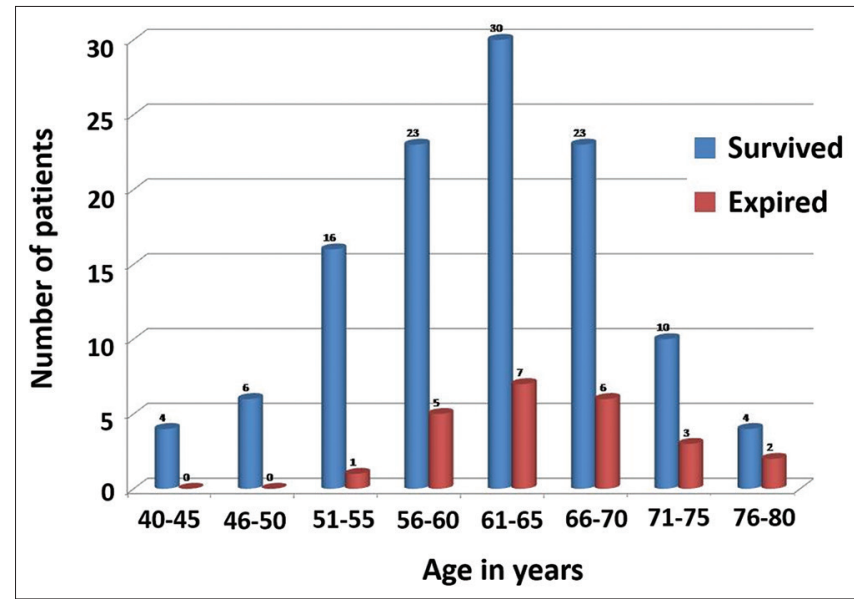

Figure 2: Age distribution of chronic obstructive pulmonary disease patients in the survived and expired group
(7.303 vs. $7.397, P<0.05)$ in the nonsurviving group while other factors such as $\mathrm{PaO}_{2}$ and $\mathrm{HCO}_{3}^{-}$were statistically insignificant. These findings suggest a poor survival if exacerbation is accompanied by a type 2 respiratory failure at the time of admission.

In COPD patients, prerenal type of renal failure is common, and COPD patients often have high urea and creatinine level than the general population. There were no significant differences $(P>0.05)$ in renal parameters between the two groups in our sample.

Electrolyte imbalance is common in COPD patients and tends to get exaggerated in acute exacerbation due to fluid retention. In our study, nonsurviving group had more severe hyponatremia as compared to the survived group (sodium 124.63 vs. $135.95 \mathrm{mEq} / \mathrm{dl}, P<0.05$ ). Other electrolytes such as serum potassium and calcium were not significantly different in both the groups.

Although polycythemia is known to occur in COPD patients due to chronic hypoxia, in developing countries like India, anemia is more common due to poor nutrition. Patients in the survived group had a mean hemoglobin $12.96 \mathrm{~g} \%$ whereas it was higher in patients of expired group with mean hemoglobin of 13.29 $\mathrm{g} \%$. The difference was not statistically significant $(P=0.716)$.

Infection is an important cause of exacerbation in COPD patients. The total leukocyte count tends to be higher in both the groups. The increase was more in nonsurviving group $\left(15,300\right.$ cells $/ \mathrm{mm}^{3}$ vs. 13,054 cells $\left./ \mathrm{mm}^{3}\right)$, but the difference was not significant statistically.

Raised blood sugar during acute phase may have a deleterious effect on the outcome. The blood sugar at the time of admission was significantly higher in the nonsurviving group (231.63 vs. $164.62 \mathrm{mg} \%, P=0.035$ ).

Liver function test was found to be deranged significantly in nonsurviving group with raised liver transaminases serum glutamic oxaloacetate transaminase (SGOT) and serum glutamic

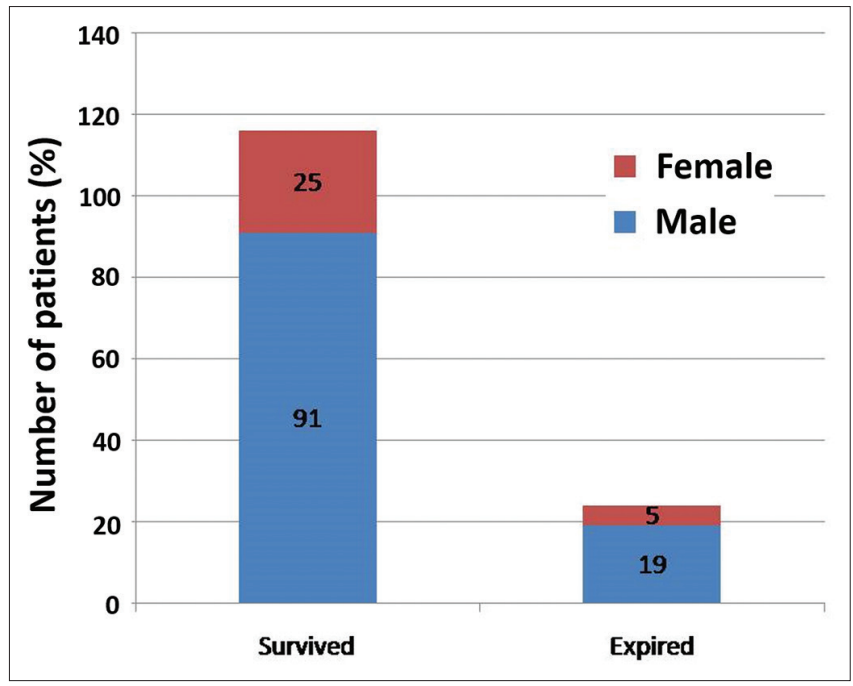

Figure 3: Gender distribution of COPD patients in each group 
Table 2: Univariate and multivariate analysis of the various acute-phase variables recorded at the time of admission Univariate analysis

Multivariate analysis

\begin{tabular}{|c|c|c|c|c|c|}
\hline \multirow[t]{2}{*}{ Variable } & \multicolumn{2}{|c|}{ Mean \pm SD } & \multirow[t]{2}{*}{$P$} & \multirow[t]{2}{*}{ OR (95\% CI) } & \multirow[t]{2}{*}{ OR (95\% Cl) } \\
\hline & Survived ( $n=116)$ & Nonsurvived $(n=24)$ & & & \\
\hline Age (years) & $61.28 \pm 8.267$ & $63.50 \pm 0.48$ & 0.219 & $1.036(0.979-1.097)$ & \\
\hline $\mathrm{PO}_{2}(\mathrm{mmHg})$ & $57.52 \pm 16.74$ & $55.88 \pm 16.5$ & 0.659 & $0.994(0.968-1.021)$ & \\
\hline $\mathrm{PCO}_{2}(\mathrm{mmHg})$ & $53.23 \pm 15.25$ & $72.09 \pm 33.1$ & 0.000 & $1.043(1.021-1.067)$ & $1.067(0.993 \pm 1.146)$ \\
\hline $\mathrm{pH}$ & $7.39 \pm 0.07$ & $7.30 \pm 0.1$ & 0.000 & $0.000(0.000-0.001)$ & $0.000(0.000 \pm 0.584)$ \\
\hline $\mathrm{HCO}_{3}^{-}(\mathrm{mmol} / \mathrm{L})$ & $32.16 \pm 8.9$ & $34.7 \pm 12.7$ & 0.238 & $1.028(0.98-1.075)$ & \\
\hline $\mathrm{SO}_{2}(\%)$ & $85.99 \pm 9.8$ & $78.5 \pm 15.6$ & 0.005 & $0.95(0.92-0.985)$ & \\
\hline Total protein (mg) (\%) & $5.93 \pm 0.63$ & $6.4 \pm 0.89$ & 0.005 & $2.485(1.314-4.699)$ & \\
\hline Albumin (mg) (\%) & $3.42 \pm 0.33$ & $3.18 \pm 0.34$ & 0.002 & $0.095(0.021-0.436)$ & \\
\hline SGOT (IU/L) & $46.12 \pm 36.9$ & $89.17 \pm 78.2$ & 0.001 & $1.015(1.006-1.025)$ & \\
\hline SGPT (IU/L) & $39.28 \pm 28.37$ & $64.46 \pm 49.2$ & 0.004 & $1.017(1.005-1.028)$ & $1.032(1.006 \pm 1.059)$ \\
\hline Urea (mg) (\%) & $62.7 \pm 20.17$ & $67.7 \pm 22.7$ & 0.519 & $1.004(0.992-1.016)$ & \\
\hline Creatinine (mg) (\%) & $1.46 \pm 0.63$ & $1.42 \pm 0.44$ & 0.782 & $0.898(0.418-1.927)$ & \\
\hline Sodium $(\mathrm{mmol} / \mathrm{L})$ & $135.95 \pm 6.1$ & $124.63 \pm 10.8$ & 0.000 & $0.826(0.762-0.894)$ & $0.779(0.689 \pm 0.881)$ \\
\hline Potassium (mmol/L) & $4.42 \pm 1.02$ & $4.19 \pm 0.88$ & 0.309 & $0.787(0.496-1.248)$ & \\
\hline Hemoglobin $(\mathrm{g})(\%)$ & $12.97 \pm 2.6$ & $13.29 \pm 2.52$ & 0.716 & $1.050(0.886-1.244)$ & \\
\hline Total leucocyte count (cells/cumm) & $13054 \pm 3990$ & $15300 \pm 9715$ & 0.074 & $1.000(1.00-1.00)$ & \\
\hline Random blood sugar (mg) (\%) & $164.62 \pm 20.51$ & $231.63 \pm 64$ & 0.011 & $1.005(1.001-1.008)$ & $1.018(1.007 \pm 1.029)$ \\
\hline Total bilirubin (mg) (\%) & $1.23 \pm 0.96$ & $1.27 \pm 1.13$ & 0.860 & $1.039(0.676-1.598)$ & \\
\hline Direct bilirubin (mg) (\%) & $0.62 \pm 0.68$ & $0.50 \pm 0.27$ & 0.439 & $0.692(0.272-1.758)$ & \\
\hline
\end{tabular}

$P<0.05$ is considered statistically significant. SD: Standard deviation, CI: Confidence interval, OR: Odds ratio, SGOT: Serum glutamic oxaloacetate transaminase, SGPT: Serum glutamic pyruvate transaminase

pyruvate transaminase (SGPT) and lower serum albumin level. Albumin level may reflect the ongoing poor nutritional status of the patient while both the transaminases and the albumin derangements may show the severity of acute phase and possibly impending multiorgan dysfunction.

\section{Multivariate analysis}

All parameters which were significant on univariate analysis $\left(\mathrm{PaCO}_{2}, \mathrm{pH}\right.$, albumin, SGOT, SGPT, sodium, and random blood sugar) were analyzed by multivariate analysis [Table 2]. The model receiver operating characteristic comprising of these five parameters was correctly classifying $93.57 \%$, and area under curve above median level was 96.8\% [Figure 4]. The five factors when combined have a sensitivity of $79.18 \%$, specificity $96.55 \%$, positive predictive value $82.61 \%$, and negative predictive value of $95.73 \%$. This means that if these factors are normal at the time of admission, one can expect a good survival.

\section{Discussion}

A total of 140 patients of COPD (diagnosed based on old spirometry reports) who presented in hospital with acute exacerbation were enrolled in this study; 24 patients $(17 \%)$ expired during hospital stay and 116 patients were discharged at the end of hospital stay. In all inhospital cases of mortality, progressive respiratory failure was the primary cause of death. This observation was partially supported by study done by Connors et al. who reported an inhospital mortality of $11 \%{ }^{[6]}$

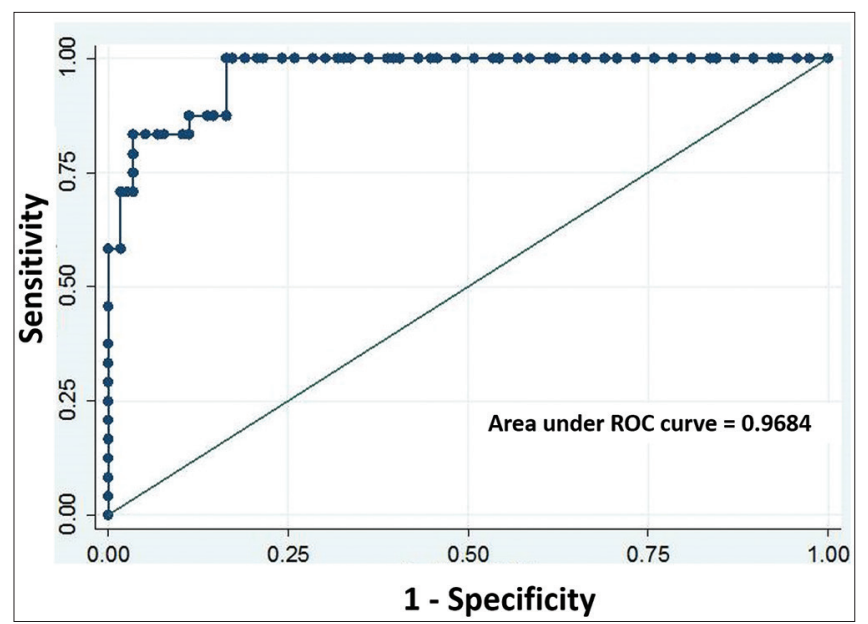

Figure 4: Model receiver operating characteristic curve comprising of the five parameters which were significant on multivariate analysis. It correctly classified the acute exacerbation in $93.57 \%$ with area under curve above median level $96.8 \%$, sensitivity of $79.18 \%$, specificity $96.55 \%$, positive predictive value $82.61 \%$, and negative predictive value $95.73 \%$

In the study done by Groenewegen et al., mortality rate was $8 \%$ during hospital stay which increased to $16 \%$ by 3 -month follow-up, $18 \%$ in 6-months follow-up, and 23\% after 1 year. ${ }^{[9]}$

Age has been shown to be an important determinant of survival in COPD patients. This finding was also seen in the present study. Age has been associated with an accelerated decline in lung function. It is important to detect and treat 
the symptoms of COPD at an earlier stage to prevent this decline. This is in accordance with the observation made by Hansen et al. ${ }^{[10]}$

In this study, five factors were seen predicting mortality in patients. These factors were $\mathrm{pH}, \mathrm{PaCO}_{2}$, sodium, random blood sugar (RBS), and SGPT (also called alanine aminotransferase or alanine transaminase).

$\mathrm{PaCO}_{2}$ is usually elevated in chronic COPD as well in AECOPD. Elevation of $\mathrm{PaCO}_{2}$ is due to alveolar hypoventilation and ventilation perfusion mismatch. Raised $\mathrm{PaCO}_{2}$ causes decrease in $\mathrm{pH}$ which is an indicator of acidosis. In chronic condition, elevated $\mathrm{PaCO}_{2}$ is usually compensated by elevation of bicarbonates so that $\mathrm{pH}$ is maintained at normal or near normal range while in acute exacerbation, there is none or only partial compensation by the kidney. Respiratory acidosis was a predictor of mortality in this study. This is in accordance with the observation made by Gray-Donald et al., ${ }^{[1]}$ but contrary to that of Connors et al. ${ }^{[6]}$

In our study, albumin was lower in nonsurviving group. Although univariate analysis showed this difference to be significant, multivariate analysis did not confirm that. This observation was contrary to the study by Khilnani et al. ${ }^{[12]}$ who concluded serum albumin in the first $24 \mathrm{~h}$ of admission to be independent predictor of mortality in patients of AECOPD. Albumin has a longer half-life of approximately 18 days, and because of this fact, it is unlikely to change with development of acute respiratory failure in patients with COPD. On the other hand, albumin is known to reflect the underlying nutritional status and to be affected by the severity of chronic illness. These factors are of obvious significance in deciding the outcome of stable COPD patients.

In our study, liver enzymes such as SGOT and SGPT were elevated significantly in expired group as compared to survived group. This difference was significant in univariate analysis while only SGPT came out as mortality predictor in multivariate analysis. Liver enzymes as mortality predictors have not been mentioned in most studies in the literature.

Renal failure in AECOPD was not found as a mortality predictor. Mean urea level was higher in expired group while serum creatinine was relatively lower but not significant statistically. This is supported by many studies such as studies by Groenewegen et al. ${ }^{[9]}$ and Seneff et al ${ }^{[13]}$. This was contrary to the study of Bhatt et al. ${ }^{[14]}$ who observed that serum creatinine at admission is an independent predictor of mortality.

Electrolyte imbalance was significant in this study. Sodium was lower in expired group as compared to survival group, and this difference was significant statistically. Chronic COPD patients most often develop pulmonary arterial hypertension followed by right heart enlargement and failure (cor pulmonale). This condition leads to fluid retention and causes hyponatremia. However, there are conflicting reports in the literature. ${ }^{[9,12-14]}$
Hyperglycemia at the time of admission is one of the mortality predictors in AECOPD. Previous studies had not commented on random blood sugar at the time of admission as mortality factor. Diabetes is one of the important comorbidities in cases of COPD. Although patients who were known diabetics were excluded in this study, previous studies have showed that patients with diabetes are more prone to get secondary infection, an important etiological factor for acute exacerbations in stable COPD patients. Patients with diabetes also have high incidence of coronary artery disease which is also an important cause of mortality in patients of COPD. Mannino et al. ${ }^{[15]}$ and Yeh et al. ${ }^{[16]}$ in their studies show that there is an increased prevalence of diabetes among COPD patients and that diabetes is independently associated with reduced lung function.

There were no significant difference in hemoglobin and total leukocyte count level in both groups. In both groups, total count was raised which indicates that infection is the most common cause of exacerbation in patients of COPD. Ittyachen et al. ${ }^{[17]}$ had found anemia to be a mortality predictor in AECOPD in rural setting.

This study has been capable of identifying some statistically competent acute-phase variables predicting the inhospital mortality of patients presenting with AECOPD patients. Limitations to get the variables into a prediction tool include small sample size and exclusion of known diabetics. We strongly feel that a multicentric study with sufficient number of patients must be further undertaken to have a definite guideline for triage and appropriateness of acute care.

\section{Conclusion}

$\mathrm{PaCO}_{2}$, arterial $\mathrm{pH}$, serum sodium level, random blood sugar, and SGPT levels measured at the time of admission are important predictors of mortality in AECOPD. All these five parameters combined together have a specificity of $96.5 \%$ and sensitivity of $80 \%$. Hence, these can be used to predict and prognosticate in terms of inpatient mortality.

\section{Financial support and sponsorship}

Nil.

\section{Conflicts of interest}

There are no conflicts of interest.

\section{ReFERENCES}

1. From the Global Strategy for the Diagnosis, Management and Prevention of COPD, Global Initiative for Chronic Obstructive Lung Disease; 2017. Available from: http://www.goldcopd.org. [Last accessed on 2018 Feb 19].

2. Mathers CD, Loncar D. Projections of global mortality and burden of disease from 2002 to 2030. PLoS Med 2006;3:e442.

3. Spencer S, Calverley PM, Burge PS, Jones PW. Impact of preventing exacerbations on deterioration of health status in COPD. Eur Respir J 2004;23:698-702.

4. Kanner RE, Anthonisen NR, Connett JE; Lung Health Study Research Group. Lower respiratory illnesses promote FEV(1) decline in current smokers but not ex-smokers with mild chronic obstructive pulmonary 
disease: Results from the lung health study. Am J Respir Crit Care Med 2001; 164:358-64.

5. Wouters EF. The burden of COPD in the Netherlands: Results from the confronting COPD survey. Respir Med 2003;97 Suppl C: S51-9.

6. Connors AF Jr., Dawson NV, Thomas C, Harrell FE Jr., Desbiens N, Fulkerson WJ, et al. Outcomes following acute exacerbation of severe chronic obstructive lung disease. The SUPPORT investigators (Study to understand prognoses and preferences for outcomes and risks of treatments) Am J Respir Crit Care Med 1996;154:959-67.

7. Steer J, Norman EM, Afolabi OA, Gibson GJ, Bourke SC. Dyspnoea severity and pneumonia as predictors of in-hospital mortality and early readmission in acute exacerbations of COPD. Thorax 2012;67:117-21.

8. Sangwan V, Chaudhry D, Malik R. Dyspnea, eosinopenia, consolidation, acidemia and atrial fibrillation score and BAP-65 score, tools for prediction of mortality in acute exacerbations of chronic obstructive pulmonary disease: A comparative pilot study. Indian J Crit Care Med 2017;21:671-7.

9. Groenewegen KH, Schols AM, Wouters EF. Mortality and mortality-related factors after hospitalization for acute exacerbation of COPD. Chest 2003; 124:459-67.

10. Hansen EF, Phanareth K, Laursen LC, Kok-Jensen A, Dirksen A. Reversible and irreversible airflow obstruction as predictor of overall mortality in asthma and chronic obstructive pulmonary disease. Am J Respir Crit Care Med 1999;159:1267-71.

11. Gray-Donald K, Gibbons L, Shapiro SH, Macklem PT, Martin JG.
Nutritional status and mortality in chronic obstructive pulmonary disease. Am J Respir Crit Care Med 1996;153:961-6.

12. Khilnani GC, Banga A, Sharma SK. Predictors of mortality of patients with acute respiratory failure secondary to chronic obstructive pulmonary disease admitted to an Intensive Care Unit: A one year study. BMC Pulm Med 2004;4:12.

13. Seneff MG, Wagner DP, Wagner RP, Zimmerman JE, Knaus WA. Hospital and 1-year survival of patients admitted to Intensive Care Units with acute exacerbation of chronic obstructive pulmonary disease. JAMA 1995;274:1852-7.

14. Bhatt SP, Mohan A, Mohan C, Mohan C, Arora S, Guleria R Predictors of in- hospital mortality and morbidity in patients with acute exacerbation of chronic obstructive pulmonary disease. Chest 2006;130:97-8.

15. Mannino DM, Thorn D, Swensen A, Holguin F. Prevalence and outcomes of diabetes, hypertension and cardiovascular disease in COPD. Eur Respir J 2008;32:962-9.

16. Yeh HC, Punjabi NM, Wang NY, Pankow JS, Duncan BB, Cox CE, et al. Cross-sectional and prospective study of lung function in adults with type 2 diabetes: The atherosclerosis risk in communities (ARIC) study. Diabetes Care 2008;31:741-6.

17. Ittyachen AM, Krishnamoorthy S, Bhatt AN, Abdulla S, Roy JO, Sugathan M, et al. Predictors of outcome in patients admitted with acute exacerbation of chronic obstructive pulmonary disease in a rural Tertiary Care Center. J Family Med Prim Care 2016;5:411-5. 